\title{
The Developmental Trajectory of Imitation in Infants with Autism Spectrum Disorders: A Prospective Study
}

\author{
Wakako Sanefuji ${ }^{1}$, Tomoka Yamamoto ${ }^{2}$ \\ ${ }^{1}$ Faculty of Human-Environmental Studies, Kyushu University, Fukuoka, Japan \\ ${ }^{2}$ Molecular Research Center for Children's Mental Development, Osaka University, Suita, Japan \\ Email: sanefuji@hes.kyushu-u.ac.jp, t-yamamoto@kokoro.med.osaka-u.ac.jp
}

Received 11 June 2014; revised 8 July 2014; accepted 4 August 2014

Copyright (C) 2014 by authors and Scientific Research Publishing Inc.

This work is licensed under the Creative Commons Attribution International License (CC BY). http://creativecommons.org/licenses/by/4.0/

cC) (7) Open Access

\begin{abstract}
Impairments in imitation are present in young children with autism spectrum disorders (ASD), but the onset of these impairments is unknown. The present study investigated the developmental trajectories affiliated with various types of imitation. Imitative performances in infancy were observed in a prospective design, and retrospectively compared between ASD and typically developing (TD) infants. The results indicated that infants who later presented the symptoms of ASD showed less imitation at 13 months of age, but not at 11 months. Both of TD and ASD infants imitated more object manipulations than gestures and meaningless movements, and gestures were more frequent than meaningless movements. Imitation impairments in ASD appear to be delayed instead of being atypical. Our findings can be used in assessments of ASD before 18 months of age.
\end{abstract}

\section{Keywords}

Imitation, Autism, Prospective Study, Infancy

\section{Introduction}

Imitation serves significant functions in early social cognitive development, including a means of communication with social partners (Nadel, Guerini, Peze, \& Rivet, 1999), a learning strategy (Uzgiris, 1981), and a foundation for shared experiences of emotions, thoughts, and intentions (Stern, 1985). In typical development (TD), imitation becomes dramatically more complex over the first 2 years of life (Kaye \& Marcus, 1981; Kuczynski, Zahn-Waxler, \& Radke-Yarrow, 1987). The development of imitation skills differs across the types of target behaviors. Imitative actions on objects emerge by 9 months (Meltzoff, 1988a); infants up to 20 months of age 
are more likely to imitate actions on objects than body movements (Abravanel, Levan-Goldschmidt, \& Stevenson, 1976; Rogdon \& Kurdek, 1977). Meaningful actions without objects, i.e., gestures, are imitated more often than non-meaningful actions without objects at 16 to 17 months of age; however, by 22 months of age, imitation of meaningful and non-meaningful actions occurs with similar frequency (McCabe \& Uzgiris, 1983).

Autism spectrum disorders (ASD), a type of neurodevelopmental disorder, are characterized by deficits in social interaction and communication skills and restricted and repetitive behaviors (American Psychiatric Association, 2000). Impaired performance on imitation tasks and other indicators have been described in children with ASD (Charman \& Baron-Cohen, 1994; Smith \& Bryson, 1994) and these persist into adulthood (Hobson \& Lee, 1999). Several studies have considered impairments in imitation as a core deficit in ASD (Rogers, Cook, \& Meryl, 2005; Williams, Whiten, \& Singh, 2004). Early impairments in imitation may affect interpersonal relationships (Rogers \& Pennington, 1991). While the findings on typical developmental course of each type of imitation have been consistent, research on the types of imitation that cause the most difficulty for children with ASD is less conclusive. Many reviews on imitation in ASD, including a meta-analysis, indicate that imitation of meaningless movements is more affected than imitation of action on objects in ASD (e.g., Colombi, Vivanti, \& Rogers, 2011; Rogers \& Williams, 2006; Williams et al., 2004): As compared to a matched control group of TD infants, young children with ASD were less likely to imitate body movements, but performed equally well on imitating object movements (e.g., Aldridge, Stone, Sweeney, \& Bower, 2000; Stone, Lemanek, Fishel, Fernandez, \& Altemeier, 1990). In contrast, however, a recent study showed that children with ASD perform less well on object imitation, but equally well in the imitation of body movements, than either TD or developmentally delayed children (e.g., Vanvuchelen, Roeyers, \& de Weerdt, 2011). Thus, there is disparity in the empirical evidence about the imitation abilities of ASD children.

One way to determine the types of imitation that may help to identify ASD is to examine imitative performance in infancy, especially during the period when imitation undergoes dramatic development. The types of imitation that children later diagnosed with ASD do not show during this period could be considered indicators of ASD. Some studies have investigated the deficits of imitation in infants with ASD through retrospective methodology including parental reports (Ornitz, Guthrie, \& Farley, 1977) or home videotapes (Receveur, Lenoir, Desombre, Roux, Barthelemy, \& Malvy, 2005). Because retrospective surveys have limitations that affect accuracy in tracking individual and group differences over time, recent studies on early identification of ASD have used a prospective methodology. To date, only one prospective study has focused on the development of imitation during the second year of life in ASD children (Young, Rogers, Hutman, Rozga, Sigman, \& Ozonoff, 2011). The study collected longitudinal data on imitation performance at 12, 18, and 24 months of age, and showed that the group of children who were diagnosed with ASD at 3 years of age were delayed in the development of imitation compared to a group of TD children at all time points. Thus, Young et al. (2011) suggest that imitation is impaired in infancy in children with ASD, but the different types of imitation (i.e., objects or body movements) were combined to form a single variable: The question about the types of imitation that are the most difficult for infants with ASD is unexplored. Additionally, in order to capture the difference in the patterns of change over time between ASD and TD, measurements during the period when imitation drastically increases are needed more frequently. Investigating imitation development according to the types of imitation at many time points in infancy could help us identify the early signs of ASD and the critical periods of delay or absence. Therefore, the aim of the present study was to investigate differences in the development of imitation between children with ASD and children with TD during infancy. Using a prospective design, we explored whether developmental trajectories differed according to the type of imitation, i.e., object manipulation, gesture, or meaningless movement, and compared the developmental paths of ASD and TD children.

\section{Method}

\subsection{Participants}

The participants were recruited through advertisements in local community publications, at posters, and in two pediatric clinics. Fifty-four infants and their families, including high-risk infants (defined as having an older sibling with ASD), were enrolled in this study. Fifteen participants were eliminated from the analysis due to missing data at one or more time points $(n=9)$, due to later emergence of ASD (having ADOS scores above ASD cut-off only at 24 months; $n=3$ ), and due to the possibility to have broad autism phenotype (having an older sibling with ASD, but ADOS scores below ASD cut-off at 16 and 24 months; $n=3$ ). Thus, the final sam- 
ple consisted of 39 infants (23 boys and 16 girls).

The participants underwent a diagnostic assessment for ASD at 16 and 24 months of age via the author-reviewed Japanese translation of the Autism Diagnostic Observation Schedule (ADOS; Lord Rutter, DiLavore \& Risi, 1999). The diagnostic stability of ASD in the second year of life through the ADOS has been consistently demonstrated in previous studies (e.g., Chawarska, Klin, Paul, \& Volkmar, 2007; Kleinman, Ventola, Pandey, Verbalis, Barton, Hodgson, Green, Dumont-Mathieu, Robins, \& Fein, 2008; Volkmar, Chawarska, \& Klin, 2005). As results, 18 infants (8 boys, 10 girls) whose scores were below the autism spectrum cut-off comprised the TD group at 16 and 24 months of age. Twenty-one infants (16 boys, 5 girls) were classified into the ASD group due to having ADOS scores above the autism spectrum cut-off at both time points; additional clinicians (licensed clinical psychologists or pediatric doctors) independently provided assessments and received clinical impressions of ASD.

\subsection{Measures and Materials}

\subsubsection{Imitation Tasks}

Three types of imitation tasks (object manipulation, gesture, and meaningless movement) were used. For all of the tasks, infants either sat on play mats in front of their primary caretakers or on their caretakers' laps. After demonstrating target imitation, the experimenter said "(Name), do this”, and infant's behaviors were observed. If an infant looked at the experimenter or caregiver and appeared to hesitate, the experimenter would encourage the infant. Encouragement without physical prompts was also given for effort; if the infant did not imitate yet, this sequence was repeated twice more so that the infant saw the modeled behaviors six additional times. Each trial was terminated if the infant imitated the modeled behavior or after $60 \mathrm{~s}$ had passed. The order of presentation of the tasks was counterbalanced across the test sessions and participants.

In the object imitation task, only a single object was available to the infant during each trial. The experimenter modeled the target manipulation three times in quick succession before handing the object to the infant. The test objects (Figure 1) were selected from the study conducted by Meltzoff (1988b). The objects and the corresponding target acts were 1) a wooden bird-shaped toy that could be set so that it produced a sound when shaken up and down; 2) an L-shaped device with a larger base connected by a hinge to a flap that could be pushed over so that it would lie flat on top of the base; 3 ) a touch panel that could be illuminated by leaning forward and touching it with the forehead; and 4) a dumbbell-shaped toy that came apart into two pieces and was put back together.

For the gesture and meaningless movement imitation tasks, the target action was demonstrated three times in succession. In this task, four meaningful gestures, selected from the study conducted by Smith and Bryson (2007), were demonstrated. The target acts were 1) "come here” (beckon with four fingers except thumb); 2) "quiet” (holding one index finger perpendicularly to lips); 3) wave goodbye; and 4) clap hands.

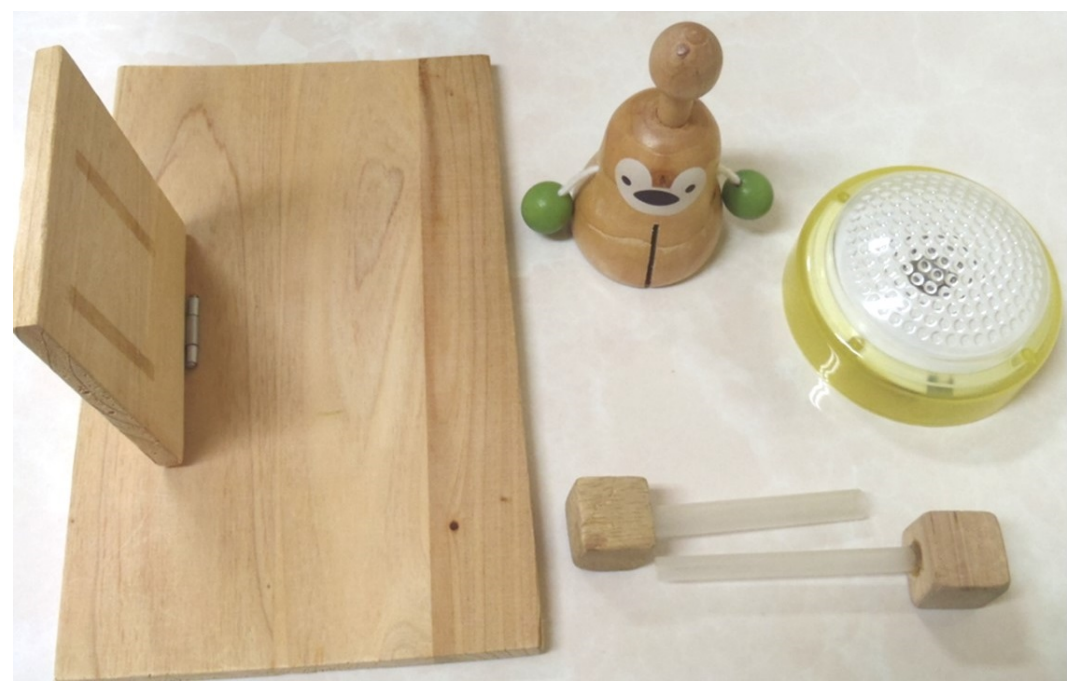

Figure 1. The four test materials used in the measures of object imitation. 
For the task of body movement imitation, four types of meaningless movements selected from previous studies (Bekkering, Wohlschläger, \& Gattis, 2000; Rogers et al., 2003) were demonstrated. The target acts were 1) open and close both hands simultaneously; 2) pat chest with one hand; 3) dangle both hands simultaneously; and 4) touch ears with both hands.

Two video cameras recorded the infant and experimenter during the administration of the tasks. The infant was assigned a score, ranging from 0 to 4 , according to the number of the four target acts he or she reproduced in each task. As a reliability check, a second observer coded $25 \%$ of the data from each task; the agreement was $100 \%$.

\subsubsection{Autism Diagnostic Observation Schedule (ADOS; Lord et al., 1999)}

The ADOS is a standardized, semi-structured behavioral observation designed to assess ASD symptoms in the domains of communication, reciprocal social interaction, play, stereotyped behaviors and restricted interests, and other abnormal behaviors. Diagnostic classification is made if the cut-off scores in communication, interaction, and the combined areas of communication and interaction are exceeded. The administration and coding of the ADOS were conducted by a trained examiner who met required reliability criteria. Because our participants were all Japanese, the present study used the author-reviewed Japanese translation for the ADOS, Western Psychological Society.

\subsection{Procedures}

The study was conducted individually in a quiet and plain room at university. The 39 participants visited university six times. During the four visits [at 11 months ( $M=11$ months 4 days), 13 months ( $M=13$ months 2 days), 15 months ( $M=15$ months 1 day), and 17 months $(M=17$ months 3 days)], imitation skills were evaluated. The diagnostic assessment of ASD, via the ADOS, was carried out at the visit when the children were 16 and 24 months old.

The caretakers of all the participants were informed about the procedure and gave their written consent for their children's participation. This study was approved by the Institutional Review Board for Clinical Research at Osaka University Hospital and was performed in accordance with the ethical standards laid down in the 1964 Declaration of Helsinki and its later amendments.

\section{Results}

Figure 2 shows the average scores observed on each imitation task for the TD and ASD groups.

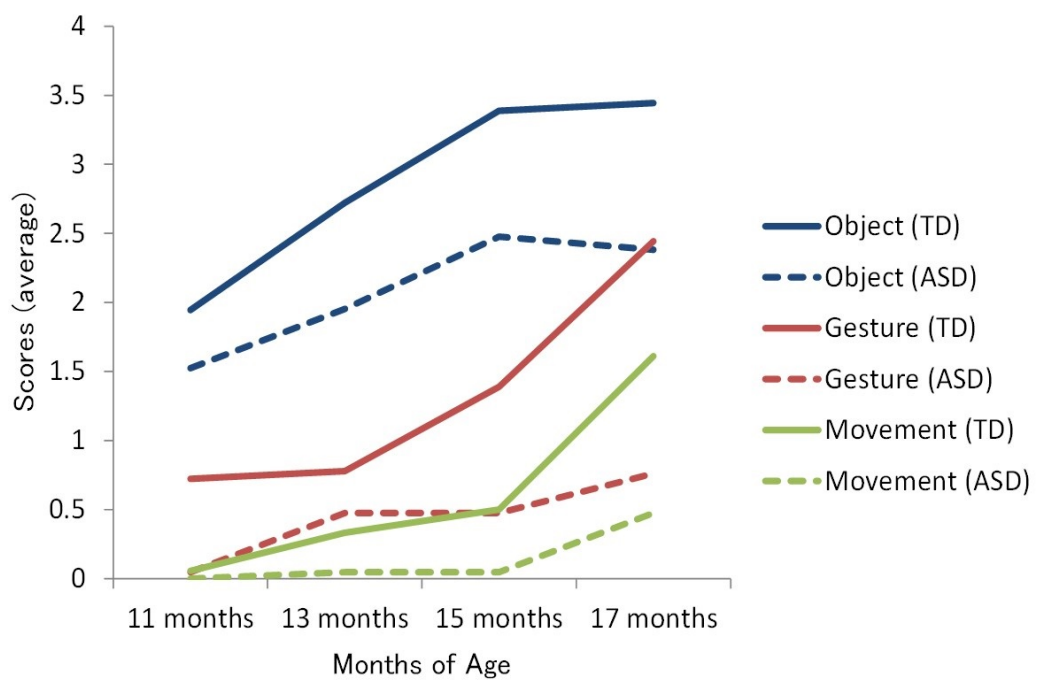

Figure 2. Developmental trajectory the three types of imitation at 11, 13, 15, and 17 months of age: object manipulation (blue line), gesture (green), and meaningless movement (red). The solid line represents the trajectory shown by the TD group and the dotted line represents that by the ASD group. 
A three-way analysis of variance (ANOVA) was conducted with the group of participants as between-subject variables and the type of imitation and age as within-subject variables. Main effects were observed for group ( $F$ $(1,37)=31.37, p<.0001)$, type of imitation $(F(2,74)=226.56, p<.0001)$, and age $(F(3,111)=4.39, p$ $=.006$ ). The TD group showed more imitation of others' behaviors compared with the ASD group. Infants imitated object manipulations more than gestures and meaningless movements $(t(74)=15.54, p<.00001 ; t(74)=$ 20.45, $p<.00001$, respectively), and more gestures than meaningless movements $(t(74)=4.91, p<.00001)$. Infants showed significant development of imitation at all measurement points; more imitation was recorded at 13 months than at 11 months $(t(111)=2.41, p=.02)$, more at 15 months than at 13 months $(t(111)=2.35, p$ $=.02)$, and more at 17 months than at 15 months $(t(111)=3.40, p=.001)$.

A significant interaction between group and age was observed $(F(3,111)=4.39, p=.006)$. The TD group demonstrated a greater amount of imitation of others' behaviors compared with the ASD group, at 13, 15, and 17 months of age $(F(1,148)=4.45, p=.04 ; F(1,148)=12.54, p=.0005 ; F(1,148)=36.40, p<.0001$, respectively), but not at 11 months $(F(1,148)=3.20, n . s$. $)$. The TD group showed significant development of imitation beginning at 13 months of age [more at 15 than at 13 months $(t(111)=2.35, p=.02)$, and more at 17 than at 15 months $(t(111)=3.61, p=.0005)]$, but there was no significant difference between 11 and 13 months of age $(t(111)=1.80, n$.s. $)$. On the other hand, a significant increase in the overall performance of imitation was observed only in the comparison of performance at 13 and 17 months of age $(t(111)=3.59, p=.0005)$ for infants with ASD.

A significant interaction between the types of imitation and age was also observed $(F(6,222)=3.60, p$ $=.002$ ). Infants showed significant development of object manipulation imitation up to 15 months of age [more at 13 months than at 11 months $(t(333)=3.11, p=.002)$ and more at 15 months than at 13 months $(t(333)=$ $3.06, p=.002)$ ], but there were no significant difference between 15 and 17 months of age $(t(333)=0.10$, n.s.). Infants showed significant development of gesture imitation between 15 and 17 months of age $(t(333)=3.45, p$ $=.0006 ; t(333)=3.96, p=.00009$, respectively). At 11 months of age, infants imitated object manipulations more than gestures and meaningless movements $(t(296)=7.65, p<.00001 ; t(296)=9.68, p<.00001$, respectively), but there was no significant difference between the imitation of gestures and meaningless movements ( $t$ $(296)=2.03$, n.s.). At 13, 15, and 17 months of age, infants imitated object manipulations more than gestures $(t$ $(296)=9.70, p<.00001 ; t(296)=11.35, p<.00001 ; t(296)=7.43, p<.00001)$ and meaningless movements $(t$ $(296)=12.18, p<.00001$; $t(296)=15.08, p<.00001 ; t(296)=10.60, p<.00001$, respectively), and more gestures than meaningless movements $(t(296)=2.48, p=.01 ; t(296)=3.74, p=.0002 ; t(296)=3.17, p=.002$, respectively).

\section{Discussion}

The differences in imitation between the infants who later presented with symptoms of ASD and the infants with TD was observed as early as 13 months of age; infants with ASD imitated less at 13 months, but not at 11 months. The present data support parental impressions about the development of their own child with ASD. Although the median age of identification of autism was 5.7 years (Shattuck, Durkin, Maenner, Newschaffer, Mandell, Wiggins, Lee, Rice, Giarelli, Kirby, Baio, Pinto-Martin, \& Cuniff, 2009), most parents have expressed concerns about developmental problems by the time the child is 18 months old (Siegel, Pliner, Eschler, \& Elliot, 1988). Our findings can be used as part of an evidence-based assessment of ASD in infants below 18 months of age. The present results also documented the developmental trajectories of imitation; infants with TD showed significant increase in imitations beginning at 13 months of age, whereas their development was slow between 11 and 13 months. Infants in the ASD group showed a relatively gradual development of imitation between 11 and 17 months of age. The identification of the periods of spontaneous development of imitation might contribute to early intervention strategies; intervention during the periods when infants with ASD show spontaneous development of imitation may lead to more profound changes, although early intervention has already been found to be associated with a good prognosis in the long term (Rogers, 1998).

The present study showed not only differences but also similarities between infants with ASD and infants with TD. The developmental trajectory shown by infants with ASD was quite similar to that shown by infants with TD. All infants imitated object manipulations more than gestures and meaningless movements, and gestures more than meaningless movements. Some studies have suggested that specific types of imitation impair- 
ments such as gesture are unique to autism (Rogers et al., 2003) although previous findings related to the types of imitation impairments in ASD have been controversial (Aldridge et al., 2000; Stone et al., 1990; Vanvuchelen et al., 2011). Other studies, however, assume that autism impairment in imitation might reflect on overall general imitation delay rather than a specific impairment (Young et al., 2011). The present results supported the latter view. The cross-sectional design of previous studies may explain the variation in the results of imitation development by age and type. The present prospective finding suggests that the developmental trajectory of imitation in ASD is delayed as compared with development in TD, rather than atypical, at least during the first half of the second year.

This study revealed the developmental trajectory of imitation in ASD by measuring developmental changes in imitation performance every other month between 11 and 17 months of age. However, in order to clarify the variety of developmental patterns of imitation in ASD, studies of the developmental trajectory of imitation in children with ASD should be extended to include children who show ASD at later time-point and to measure continuously after 17 months of age. In fact, in the case of social, language and motor development measured by the Mullen Scales of Early Learning and the Communication and Symbolic Behavior Scales Developmental Profile, impairment during the second year, e.g., from 14 to 24 months, was greater in the Early-onset ASD group than in the Later-onset ASD group, but comparable at 36 months in both groups (Landa et al., 2013). Our finding that infants who later displayed the features of ASD showed delayed development of imitation beginning at age 13 months should be extended to subsequent development. Future studies that focus on the delayed development of imitation on the posterior severity of ASD and on the subsequent development of other social cognitive skills may shed light on the development of social cognition in ASD as well as the influence of imitation on social cognition development in general. A limitation of this study was the lack of comparison to a group of non-ASD infants with other developmental delays: Young et al. (2011) reported that imitation in the High-risk group with other delays was not significantly different from imitation in the ASD group. Examining and identifying the types and/or periods of imitation that differentiated ASD from other developmental delays could be useful in establishing an early diagnostic assessment tool for ASD.

\section{Acknowledgements}

We are grateful to the infants and their families who participated in this study. We also appreciate Aya Kayanuma for her help to collect data; and Ikuko Mohri and Masako Taniike for their provisions of assistance to conducting this study. This work was supported by JSPS Grant-in-Aid for Scientific Research (A) Grant Number 23683015.

\section{References}

Abravanel, E., Levan-Goldschmidt, E., \& Stevenson, M. B. (1976). Action Imitation: The Early Phase of Infancy. Child Development, 47, 1032-1044. http://dx.doi.org/10.1111/j.1467-8624.1976.tb02284.X

Aldridge, M. A., Stone, K. R., Sweeney, M. H., \& Bower, T. G. R. (2000). Preverbal Children with Autism Understand the Intentions of Others. Developmental Science, 3, 294-301. http://dx.doi.org/10.1111/1467-7687.00123

American Psychiatric Association (2000). Diagnostic and Statistical Manual of Mental Disorders DSM-IV-TR. Washington DC: American Psychiatric Association. http://dx.doi.org/10.1080/713755872

Bekkering, H., Wohlschläger, A., \& Gattis, M. (2000). Imitation of Gestures in Children Is Goal-directed. The Quarterly Journal of Experimental Psychology Section A: Human Experimental Psychology, 53, 153-164. http://dx.doi.org/10.1080/027249800390718

Charman, T., \& Baron-Cohen, S. (1994). Another Look at Imitation in Autism. Development and Psychopathology, 6, 403-413. http://dx.doi.org/10.1017/S0954579400006015

Chawarska, K., Klin, A., Paul, R., \& Volkmar, F. (2007). Autism Spectrum Disorder in the Second Year: Stability and Change in Syndrome Expression. Journal of Child Psychology and Psychiatry, 48, 128-138. http://dx.doi.org/10.1111/j.1469-7610.2006.01685.x

Colombi, C., Vivanti, G., \& Rogers, S. J. (2011). Imitation in ASD. In D. Fein (Ed.), The Neuropsychology of Autism (pp. 243-266). New York: Oxford University Press.

Hobson, R. P., \& Lee, A. (1999). Imitation and Identification in Autism. Journal of Child Psychology and Psychiatry, 40, 649-659. http://dx.doi.org/10.1111/1469-7610.00481 
Kaye, K., \& Marcus, J. (1981). Infant Imitation: The Sensory-Motor Agenda. Developmental Psychology, 17, $258-265$. http://dx.doi.org/10.1037/0012-1649.17.3.258

Kleinman, J. M., Ventola, P. E., Pandey, J., Verbalis, A. D., Barton, M., Hodgson, S. et al. (2008). Diagnostic Stability in Very Young Children with Autism Spectrum Disorders. Journal of Autism and Developmental Disorders, 38, 606-615. http://dx.doi.org/10.1007/s10803-007-0427-8

Kuczynski, L., Zahn-Waxler, C., \& Radke-Yarrow, M. (1987). Development and Content of Imitation in the Second and Third Years of Life: A Socialization Perspective. Developmental Psychology, 23, 276-282. http://dx.doi.org/10.1037/0012-1649.23.2.276

Landa, R. J., Gross, A. L., Stuart, E. A., \& Faherty, A. (2013). Developmental Trajectories in Children with and without Autism Spectrum Disorders: The First 3 Years. Child Development, 84, 429-442. http://dx.doi.org/10.1111/j.1467-8624.2012.01870.x

Lord, C., Rutter, M., Di Lavore, P., \& Risi, S. (1999). Autism Diagnostic Observation Schedule. Los Angeles, CA: Western Psychological Services.

McCabe, M., \& Uzgiris, I. C. (1983). Effects of Model and Action on Imitation in Infancy. Merrill-Palmer Quarterly, 29, 69-82.

Meltzoff, A. N. (1988a). Infant Imitation and Memory: Nine-Month-Olds in Immediate and Deferred Tests. Child Development, 59, 217-225. http://dx.doi.org/10.1111/j.1467-8624.1988.tb03210.x

Meltzoff, A. N. (1988b). Infant Imitation after a 1-Week Delay: Long-Term Memory for Novel Acts and Multiple Stimuli. Developmental Psychology, 24, 470-476. http://dx.doi.org/10.1037/0012-1649.24.4.470

Nadel, J., Guerini, C., Peze, A., \& Rivet, C. (1999). The Evolving Nature of Imitation as a Format for Communication. In J. Nadel, \& G. Butterworth (Eds.), Imitation in Infancy (pp. 209-234). Cambridge: Cambridge University Press.

Ornitz, E. M., Guthrie, D., \& Farley, A. H. (1977). The Early Development of Autistic Children. Journal of Autism and Developmental Disorders, 7, 207-229. http://dx.doi.org/10.1007/BF01538999

Receveur, C., Lenoir, P., Desombre, H., Roux, S., Barthelemy, C., \& Malvy, J. (2005). Interaction and Imitation Deficits from Infancy to 4 Years of Age in Children with Autism. Autism, 9, 69-82. http://dx.doi.org/10.1177/1362361305049030

Rogdon, M. M., \& Kurdek, L. (1977). Vocal and Gestural Imitation in 8-, 14-, and 20-Month-Old Children. The Journal of Genetic Psychology, 131, 115-123. http://dx.doi.org/10.1080/00221325.1977.10533280

Rogers, S. (1998). Empirically Supported Comprehensive Treatments for Young Children with Autism. Journal of Clinical Child Psychology, 27, 168-179. http://dx.doi.org/10.1207/s15374424jccp2702_4

Rogers, S. J., Cook, I., \& Meryl, A. (2005). Imitation and Play in Autism. In F. R. Volkmar, R. Paul, A. Klin, \& D. Cohen (Eds.), Handbook of Autism and Pervasive Developmental Disorder (pp. 382-405). Hoboken, NJ: John Wiley \& Sons, Inc.

Rogers, S. J., Hepburn, S. L., Stackhouse, T., \& Wehner, E. (2003). Imitation Performance in Toddlers with Autism and Those with Other Developmental Disorders. Journal of Child Psychology and Psychiatry, 44, 763-781. http://dx.doi.org/10.1111/1469-7610.00162

Rogers, S. J., \& Pennington, B. F. (1991). A Theoretical Approach to the Deficits in Infantile Autism. Development and Psychopathology, 3, 137-162. http://dx.doi.org/10.1017/S0954579400000043

Rogers, S. J., \& Williams, J. G. (2006). Imitation and the Social Mind: Autism and Typical Development. New York: The Guilford Press.

Shattuck, P. T., Durkin, M., Maenner, M., Newschaffer, C., Mandell, D. S., Wiggins, L. et al. (2009). Timing of Identification among Children with an Autism Spectrum Disorder: Findings from a Population-Based Surveillance Study. Journal of the American Academy of Child and Adolescent Psychiatry, 48, 474-483. http://dx.doi.org/10.1097/CHI.0b013e31819b3848

Siegel, B., Pliner, C., Eschler, J., \& Elliot, G. R. (1988). How Children with Autism are Diagnosed: Difficulties in Identification of Children with Multiple Developmental Delays. Journal of Developmental and Behavioral Pediatrics, 9, $199-204$. http://dx.doi.org/10.1097/00004703-198808000-00004

Smith, I. M., \& Bryson, S. E. (1994). Imitation and Action in Autism: A Critical Review. Psychological Bulletin, 116, 259273. http://dx.doi.org/10.1037/0033-2909.116.2.259

Smith, I. M., \& Bryson, S. E. (2007). Gesture Imitation in Autism: II. Symbolic Gestures and Pantomimed Object Use. Cognitive Neuropsychology, 24, 679-700. http://dx.doi.org/10.1080/02643290701669703

Stern, D. N. (1985). The Interpersonal World of the Human Infant. New York: Basic Books.

Stone, W. L., Lemanek, K. L., Fishel, P. T., Fernandez, M. C., \& Altemeier, W. A. (1990). Play and Imitation Skills in the Diagnosis of Autism in Young Children. Pediatrics, 86, 267-272.

Uzgiris, I. C. (1981). Two Functions of Imitation during Infancy. International Journal of Behavioral Development, 4, 1-12. http://dx.doi.org/10.1177/016502548100400101 
Vanvuchelen, M., Roeyers, H., \& De Weerdt, W. (2011). Imitation Assessment and Its Utility to the Diagnosis of Autism: Evidence from Consecutive Clinical Preschool Referrals for Suspected Autism. Journal of Autism and Developmental Disorders, 41, 484-496. http://dx.doi.org/10.1007/s10803-010-1074-z

Volkmar, F., Chawarska, K., \& Klin, A. (2005). Autism in Infancy and Early Childhood. Annual Review of Psychology, 56, 315-336. http://dx.doi.org/10.1146/annurev.psych.56.091103.070159

Williams, J. H. G., Whiten, A., \& Singh, T. (2004). A Systematic Review of Action Imitation in Autistic Spectrum Disorder. Journal of Autism and Developmental Disorders, 34, 285-299. http://dx.doi.org/10.1023/B:JADD.0000029551.56735.3a

Young, G. S., Rogers, S. J., Hutman, T., Rozga, A., Sigman, M., \& Ozonoff, S. (2011). Imitation from 12 to 24 Months in Autism and Typical Development: A Longitudinal Rasch Analysis. Developmental Psychology, 47, 1565-1578. http://dx.doi.org/10.1037/a0025418 
Scientific Research Publishing (SCIRP) is one of the largest Open Access journal publishers. It is currently publishing more than 200 open access, online, peer-reviewed journals covering a wide range of academic disciplines. SCIRP serves the worldwide academic communities and contributes to the progress and application of science with its publication.

Other selected journals from SCIRP are listed as below. Submit your manuscript to us via either submit@scirp.org or Online Submission Portal.
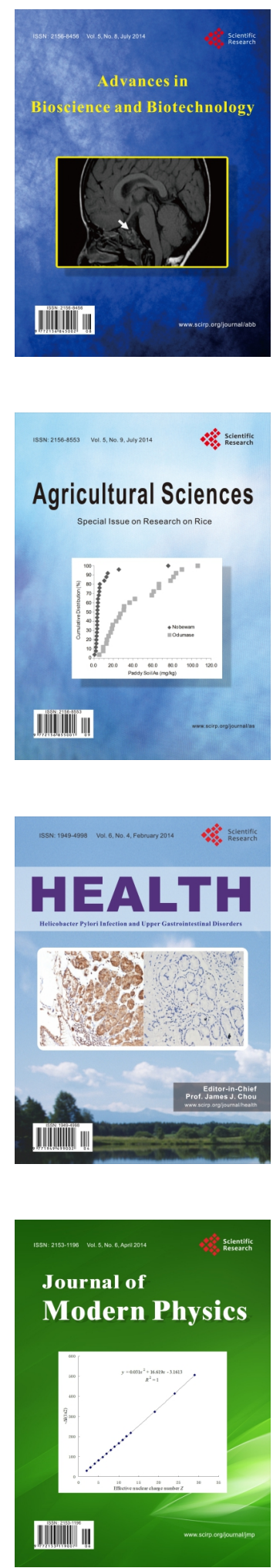
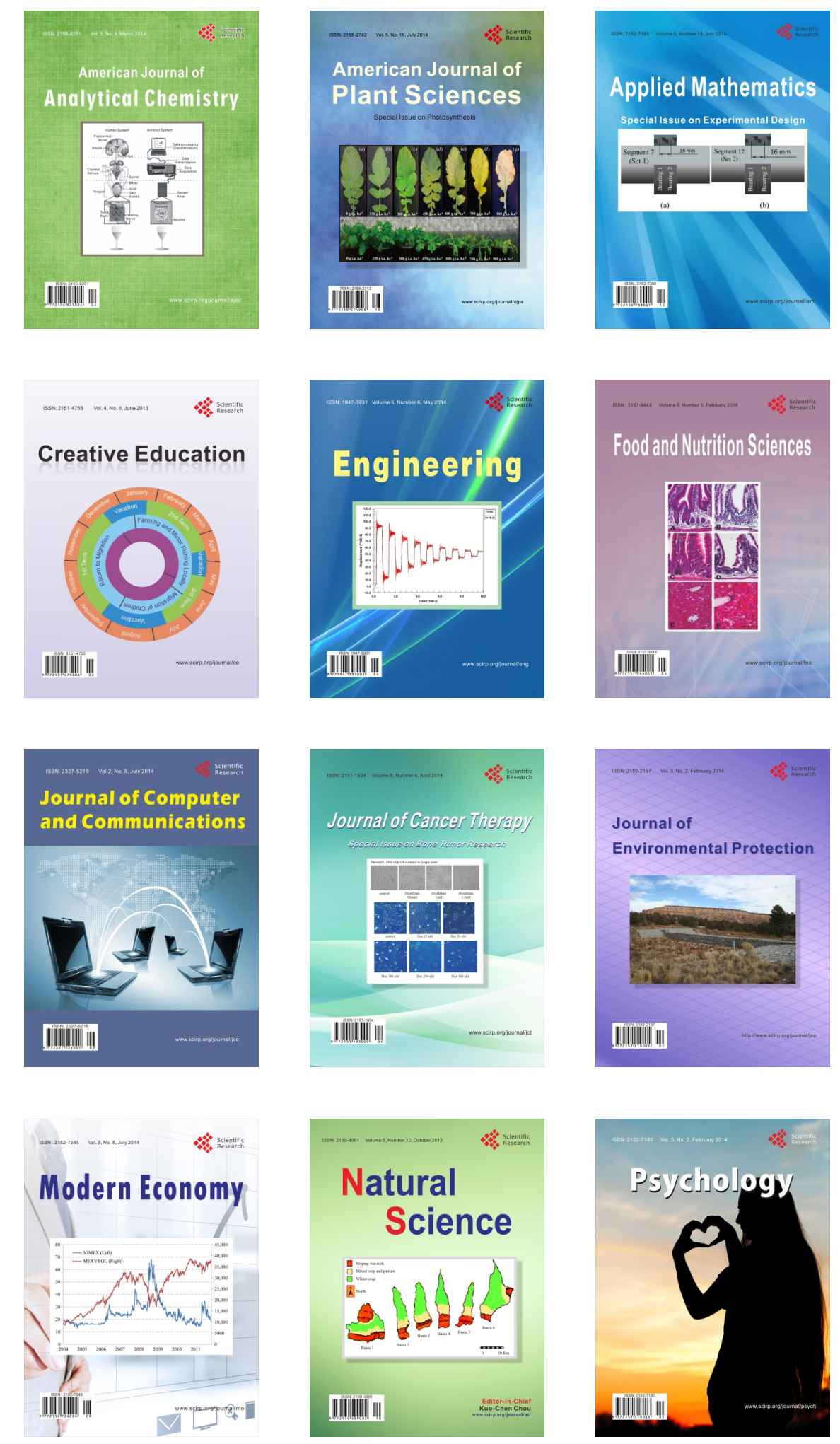